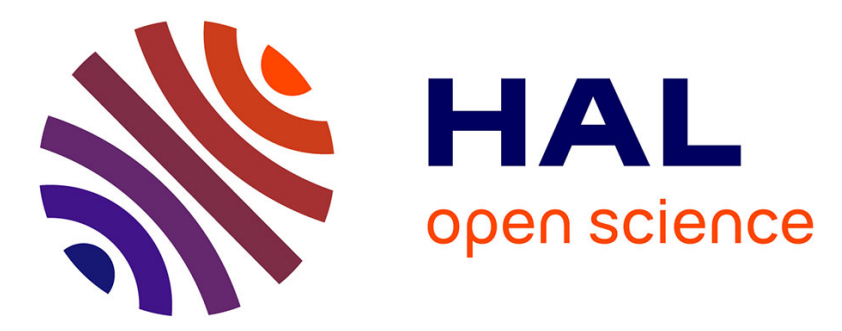

\title{
Validity of diagnostic criteria for chronic inflammatory demyelinating polyneuropathy: a multicentre European study.
}

Yusuf A. Rajabally, Guillaume Nicolas, Francoise Pieret, Pierre Bouche, Peter y K van den Bergh

\section{To cite this version:}

Yusuf A. Rajabally, Guillaume Nicolas, Francoise Pieret, Pierre Bouche, Peter y K van den Bergh. Validity of diagnostic criteria for chronic inflammatory demyelinating polyneuropathy: a multicentre European study.. Journal of Neurology, Neurosurgery and Psychiatry, 2009, 80 (12), pp.1364. 10.1136/jnnp.2009.179358 . hal-00552786

\section{HAL Id: hal-00552786 https://hal.science/hal-00552786}

Submitted on 6 Jan 2011

HAL is a multi-disciplinary open access archive for the deposit and dissemination of scientific research documents, whether they are published or not. The documents may come from teaching and research institutions in France or abroad, or from public or private research centers.
L'archive ouverte pluridisciplinaire HAL, est destinée au dépôt et à la diffusion de documents scientifiques de niveau recherche, publiés ou non, émanant des établissements d'enseignement et de recherche français ou étrangers, des laboratoires publics ou privés. 


\title{
Validity of diagnostic criteria for chronic inflammatory demyelinating polyneuropathy: a multicentre European study.
}

\author{
Yusuf A. Rajabally, ${ }^{1} \quad$ Guillaume Nicolas, ${ }^{2,3} \quad$ Françoise Piéret, $^{4}$ \\ Pierre Bouche, ${ }^{3} \quad$ Peter Y.K. Van den Bergh. ${ }^{4}$
}

1: Neuromuscular Clinic, Department of Neurology, University Hospitals of Leicester, Leicester, U.K.

2: Centre de Référence Maladies Neuromusculaires de l'Enfant et de l'Adulte Nantes-Angers, Centre Hospitalier Universitaire d'Angers, Angers, France. 3: Fédération de Neurophysiologie Clinique, Hôpital Salp $\square$ trière, Paris, France. 4: Centre de Référence Neuromusculaire, Service de Neurologie, Cliniques Universitaires St-Luc, Université Catholique de Louvain, Brussels, Belgium.

Key words: EFNS/PNS Guidelines; chronic inflammatory demyelinating polyneuropathy; electrophysiology; sensitivity; specificity.

Word Count: 3542

The Corresponding Author has the right to grant on behalf of all authors and does grant on behalf of all authors, an exclusive licence (or non-exclusive for government employees) on a worldwide basis to the BMJ Publishing Group Ltd and its Licensees to permit this article (if accepted) to be published in JNNP and any other BMJPGL products to exploit all subsidiary rights, as set out in our licence.

Correspondence to:

Dr. Yusuf A. Rajabally

Neuromuscular Clinic

Department of Neurology

University Hospitals of Leicester

Leicester General Hospital

Leicester LE5 4PW

United Kingdom.

Tel: +44 1162584574

Fax: +44 1162584875

E-mail: yusuf.rajabally@uhl-tr.nhs.uk 


\section{$\underline{\text { Abstract. }}$}

Background: Diagnostic criteria for chronic inflammatory demyelinating polyneuropathy (CIDP) have variable sensitivity and specificity. Newly published criteria by Koski et al. [2009] combine clinical and electrophysiological components, either of which suffices to establish the diagnosis. European Federation of Neurological Societies/Peripheral Nerve Society (EFNS/PNS) criteria [2006], require mandatory electrophysiology, as do other sets of criteria.

Methods: We assessed the value of the two above-mentioned sets of criteria, on 151 patients with CIDP, and 162 controls with axonal neuropathy, from four European centres. Results were compared with Van den Bergh and Piéret's criteria [2004], and those of the American Academy of Neurology (AAN) [1991]. The utility of more extensive nerve conduction studies was ascertained.

Results: Koski et al.'s criteria had a sensitivity of $63 \%$ and specificity of $99.3 \%$. With unilateral, right-sided, forearm/foreleg, four-nerve studies, EFNS/PNS criteria offered a sensitivity of $81.3 \%$ and specificity of $96.2 \%$ for "definite/probable" CIDP. Van den Bergh and Piéret's criteria had a sensitivity of $79.5 \%$ and specificity of 96.9\%. AAN criteria were poorly sensitive (45.7\%) but highly specific (100\%). "Possible" electrophysiological CIDP as per EFNS/PNS criteria were poorly specific (69.2\%). More extensive studies increased diagnostic sensitivity of EFNS/PNS criteria $(96.7 \%)$ but reduced specificity $(79.3 \%)$.

Conclusions: In our patient populations, the EFNS/PNS criteria were the most sensitive and allowed identifying a highly significantly greater number of patients than Koski et al.'s criteria. The latter were comparable in specificity to the “definite/probable" EFNS/PNS electrodiagnostic subcategories. More extensive nerve conduction studies improved diagnostic yield but resulted in loss of specificity. 


\section{Introduction.}

Chronic inflammatory demyelinating polyneuropathy (CIDP) is a clinically heterogeneous disorder. In clinical practice, diagnosis relies heavily on electrophysiology, but electrodiagnostic criteria lack sensitivity [1-4]. This may result in misdiagnosis, potentially denying patients effective treatment.

A new set of criteria comprising clinical and electrophysiological components has been reported and was validated in 48 patients by Koski et al. [5] These diagnostic criteria represent the only ones reported so far for which either clinical or electrophysiological requirements, are sufficient to establish a diagnosis of CIDP. Their reliability has not so far been ascertained in other larger CIDP patient populations. Guidelines published in 2006 from the European Federation of Neurological Societies/Peripheral Nerve Society (EFNS/PNS) had also defined clinical and electrodiagnostic criteria. [1] The electrophysiological component within these criteria, essential for establishing the diagnosis, was based on a set originally described by Van den Bergh and Piéret. [2] Distal compound muscle action potential duration prolongation, found in one study to be a highly sensitive independent marker of CIDP, [6] was used as an additional criterion to those proposed by Van den Bergh and Piéret, to produce the EFNS/PNS electrodiagnostic criteria. A further electrodiagnostic subcategory ("possible CIDP”) was also added, encompassing cases with demyelinating features in a single nerve instead of in 2 nerves. Subsequently, high diagnostic sensitivity and high negative and positive predictive values of the EFNS/PNS criteria were demonstrated in one population of CIDP patients. [7] This has not been confirmed however more widely, in different populations. The American 
Academy of Neurology (AAN) criteria [8] also require mandatory electrophysiology for the diagnosis.

We here evaluate the sensitivity and specificity of Koski et al.'s criteria [5] (Table 1) and the EFNS/PNS criteria [1] (Table 2), in a study involving four European Neurology/Neurophysiology centres. Direct evaluation of the electrophysiological part of these criteria was performed on patients meeting EFNS/PNS clinical requirements, as electrophysiology is mandatory for diagnosis with these criteria. Comparison of nerve conduction studies of varying extensiveness was also made, as was comparison with the sensitivity and specificity of the electrophysiological part of the American Academy of Neurology (AAN) criteria, [8] also essential for the diagnosis (Table 3). ). We also aimed to evaluate the diagnostic value of distal compound muscle action potential (CMAP) duration prolongation [6] by comparing the EFNS/PNS electrophysiological criteria with the original criteria of Van den Bergh and Piéret [2], which did not include this parameter. 
$\underline{\text { Table 1: Koski et al.'s criteria for chronic inflammatory demyelinating polyneuropathy (CIDP) }}$

[5]:

Patients with a chronic polyneuropathy, progressive for at least 8 weeks, would be classified as having CIDP if they had:

No serum paraprotein and

No documented genetic abnormality

\section{AND EITHER (electrophysiological component):}

a) at least $75 \%$ of motor nerves tested has a recordable response AND one of the following conditions is satisfied:

(i) more than $50 \%$ of the motor nerves tested had an abnormal distal latency, ${ }^{1}$ or

(ii) more than $50 \%$ of the motor nerves tested had abnormal conduction velocity, ${ }^{1}$ or

(iii) more than $50 \%$ of the motor nerves tested had abnormal F-latency. ${ }^{1}$

\section{$\underline{\text { OR (clinical component): }}$}

b) (i) Symmetric onset or symmetric exam, and

(ii) Weakness in all four limbs, and

(iii) At least one limb with proximal weakness.

1: As per AAN criteria (see Table 3) 


\section{Table 2: EFNS/PNS electrodiagnostic criteria for CIDP [1]:}

I: "Definite CIDP": at least one of the following:

A. At least $50 \%$ prolongation of the motor distal latency above the upper limit of normal values in two nerves, or

B. At least $30 \%$ reduction of motor conduction velocity below the lower limit of normal values in two nerves, or

C. At least $20 \%$ prolongation of F-wave latency above the upper limit of normal values in two nerves ( $>50 \%$ if amplitude of negative peak compound muscle action potential (CMAP) $<80 \%$ of lower limit of normal values), or

D. Absence of $\mathrm{F}$ waves in two nerves if these nerves have amplitudes of distal negative peak at least 20

$\%$ of lower limit of normal values + at least one other demyelinating parameter in at least one other nerve, or

E. Partial motor conduction block: at least $50 \%$ amplitude reduction of the proximal negative peak CMAP relative to distal, if distal negative peak CMAP at least $20 \%$ of lower limit of normal values, in two nerves, or in one nerve + at least one other demyelinating parameter in at least one other nerve, or F: Abnormal temporal dispersion (> $30 \%$ duration increase between proximal and distal negative peak CMAP) in at least two nerves, or

G. Distal CMAP duration (interval between onset of the first negative peak and return to baseline of last negative peak) of at least $9 \mathrm{~ms}$ in at least one nerve nerve + at least one other demyelinating parameter in at least one other nerve.

\section{II. "Probable CIDP"}

At least $30 \%$ amplitude reduction of the proximal negative peak CMAP relative to distal, excluding the posterior tibial nerve,, if distal negative peak CMAP at least $20 \%$ of lower limit of normal values in two nerves, or in one nerve nerve + at least one other demyelinating parameter in at least one other nerve.

\section{III. "Possible CIDP"}

As in "I" but in only one nerve. 


\section{Table 3: AAN electrodiagnostic criteria for CIDP [8]:}

At least three of the following four to be fulfilled:

1. Significant reduction of motor nerve conduction velocity in 2 or more motor nerves:

a. $<80 \%$ of LLN if CMAP $>80 \%$ of LLN

b. $\quad<70 \%$ of LLN if CMAP $<80 \%$ of LLN

2. Partial conduction block or abnormal temporal dispersion in 1 or more motor nerves: either peroneal nerve between ankle and below fibular head, median nerve between wrist and elbow, or ulnar nerve between wrist and below elbow.

Criteria suggestive of partial conduction block: $<15 \%$ change in duration between proximal and distal sites and $>20 \%$ drop in negative peak (-p) area or peak-to-peak (p-p) amplitude between proximal and distal sites.

Criteria for abnormal temporal dispersion and possible conduction block: $>15 \%$ change in duration between proximal and distal sites and $>20 \%$ drop in $-\mathrm{p}$ area or p-p amplitude between proximal and distal sites

3. Significant prolongation of distal motor latency in 2 or more motor nerves:

a. $\quad>125 \%$ of ULN if CMAP $>80 \%$ of LLN

b. $>150 \%$ of ULN if CMAP $<80 \%$ of LLN

4. Significant prolongation or absence of F-waves in 2 or more motor nerves:

a. $\quad>120 \%$ of ULN if CMAP $>80 \%$ of LLN

b. $>150 \%$ of ULN if CMAP $<80 \%$ of LLN 


\section{Materials and Methods.}

From the four participating centres, Leicester (U.K.), Paris (France), Angers (France) and Brussels (Belgium), patients fulfilling clinical criteria defined by EFNS/PNS Guidelines for CIDP, were included, and their clinical and electrophysiological records were reviewed. Patients were selected on the basis of a clinical diagnosis of typical CIDP, as defined by the EFNS/PNS Guidelines. In addition, as per these Guidelines which define atypical forms, we included patients with Lewis-Sumner syndrome, predominantly distal weakness, focal involvement and those with associated concomitant diseases: diabetes mellitus, $\operatorname{IgG}$ and $\operatorname{IgA}$ monoclonal gammopathy, and IgM monoclonal gammopathy without anti-MAG activity. All exclusion criteria, as listed by EFNS/PNS Guidelines were otherwise met. [1] The patients from Brussels were those from which the Van den Bergh and Piéret criteria were originally derived. This did not affect the results for the EFNS/PNS criteria, as these patients were excluded from this analysis. The study was reviewed by our relevant Institutional Boards and did not require Ethics Commitee approval.

In view of the retrospective design, electrophysiological studies had been performed in a non-standardized manner, the number of nerves studied having varied from 4 to 8. Studies had been performed in the majority of patients pre-treatment, although in some cases, due to technical issues and lack of sufficiently extensive earlier evaluations (less than 4 motor nerves adequately tested), a subsequent, post-treatment study was considered for the current analysis. Extensiveness of the study of arm nerves varied from the distal forearm segment only, to a full length study up to Erb's point. The CMAPs were evoked from the median nerve (stimulating at wrist, elbow, 
and in some, axilla, Erb's point, and recording at the Abductor Pollicis Brevis muscle), ulnar nerve (stimulating at wrist, below elbow, and in some, above elbow, axilla, Erb's point, and recording at the Abductor Digiti Minimi muscle), common peroneal nerve (stimulating at ankle and fibular neck and recording at the Extensor Digitorum Brevis muscle) and tibial nerve (stimulating at ankle and popliteal fossa and recording at the Abductor Hallucis muscle). All nerve conductions were performed at ankle temperature $\geq 30^{\circ} \mathrm{C}$ and palm temperature $\geq 33^{\circ} \mathrm{C}$. [1] Results were analyzed with each laboratory's range of normal values, and presence of demyelinating range values determined for each relevant parameter. Fulfillment of each set of electrodiagnostic criteria for CIDP was ascertained in each case. For consideration with proximal studies, the EFNS/PNS "probable CIDP” subcategory was modified, redefining conduction block as at least $50 \%$ amplitude reduction in all cases (rather than 30\%), at Erb's point, for proximal upper limb studies, in accordance with previous evidence. $[9,10]$

For the criteria described by Koski et al., patients with Lewis-Sumner Syndrome or monoclonal gammopathy were excluded from analysis, as per these authors' definitions. [5] Clinical records and histories were reviewed in detail to establish fulfillment of the clinical component of these criteria. Electrophysiological criteria fulfillment was established in each case, using available distal (foream and foreleg) studies with AAN criteria cut-offs. [5, 8]

Sensitivity and specificity were ascertained with 4 different nerve conduction study protocols ("PROTOCOLS I-IV") for EFNS/PNS criteria. "PROTOCOL I" corresponded to a unilateral right-sided distal segment 4 nerve-studies, including cases of "probable" and "definite" CIDP only. [2] "PROTOCOL II" considered the same unilateral 4-nerve study, but also included cases 
of "possible CIDP." [2] "PROTOCOL III" related to nerve conduction studies of 5 to 8 motor nerves, with proximal stimulations for at least 2, excluding the cases of "possible CIDP". Finally, "PROTOCOL IV" corresponded to similar more extensive nerve conductions, but including the cases of “possible CIDP”. Fulfilment of Van den Bergh and Piéret's criteria [2] was ascertained using PROTOCOL I. AAN criteria [8] were evaluated with all available nerve conduction results (irrespective of side of study).

To determine the specificity of the different criteria, we utilized the electrophysiological data from controls suffering from distal sensory or sensori-motor axonal neuropathy, from 3 of our 4 centres (Leicester, Paris and Brussels). None fulfilled EFNS/PNS clinical criteria for typical CIDP. All had been investigated with nerve conduction studies evaluating at least 4 motor nerves in the forearm and foreleg segments. A proportion had been investigated more extensively, with 5 to 8 nerves studied, and at least 2 upper limb nerves tested in their proximal segments.

The comparative diagnostic gain in sensitivity, achieved with the use of the more sensitive set of criteria, was calculated using a Mc Nemar's Test. 


\section{Results.}

We included 54 patients from Leicester, U.K., 40 patients from Paris, France, 29

patients from Angers, France, and 28 patients from Brussels, Belgium, totalling 151

patients. Patients with CIDP had a mean age at onset of 52.5 years (mean for

Leicester: 56.5; for Paris: 49.0; for Angers: 51.8; for Brussels: 50.4). There were 95

males and 56 females. Seven of our 151 patients had not been treated with

immunomodulatory therapy, due to mild disease. Of the 144 who were treated, 136

(94.4\%) had responded to treatment. Of the 108 CIDP patients who had a documented

lumbar puncture result, 83 (76.9\%), had a raised cerebrospinal fluid protein level. We utilized a total of 162 controls with chronic distal sensori-motor axonal neuropathy. Thirty-five were recruited from Paris, who had been investigated for 5-8 nerves with proximal stimulations for at least 2 . Ninety-five controls were recruited from Leicester. Of those, 47 had been investigated for 5 to 8 nerves, with proximal stimulations for at least 2, and 48 had only undergone the basic unilateral right-sided 4-nerve distal segment study. Thirty-two controls were recruited from Brussels, all having undergone distal segment four-nerve studies. All controls were used to determine the specificity of AAN [8] and Van den Bergh and Piéret's [2] criteria. Controls from Brussels were not included in the calculation of specificity of EFNS/PNS criteria, as their data did not include distal CMAP duration. Only 22 of the controls from Brussels were used to determine the specificity of Koski et al.'s criteria, as detailed clinical records were required for that purpose, and were unavailable for 10. Controls were older than CIDP patients (mean age: 61.2 years) and consisted of 93 males and 69 females. 
The results for sensitivity are summarized in Table 3 . Those relating to specifity are detailed in Table 4. Koski et al.'s criteria [5] offered a sensitivity of $63 \%$ and specificity of $99.3 \%$ versus controls. The total number of CIDP patients evaluated for these criteria was 127, excluding as per their definition, cases of Lewis-Sumner syndrome and those associated with monoclonal gammopathy. Of the cases meeting Koski et al.'s criteria, 30 fulfilled both clinical and electrophysiological components, 25 fulfilled only clinical criteria, and 25 met only the electrophysiological requirements. The criteria were highly specific $(99.3 \%)$.

Only CIDP patients from Leicester, Paris and Angers were evaluated for distal CMAP duration prolongation, and had more extensive nerve conduction studies. Hence, the sensitivity of the EFNS/PNS electrophysiological criteria for CIDP [1] in those 3 centres combined was $81.3 \%$, and specificity was $96.2 \%$ using PROTOCOL I. With PROTOCOL II, which also included the "possible CIDP" cases, sensitivity improved to $96.7 \%$. However, this also resulted in increased misdiagnosis of controls as having CIDP, thereby reducing specificity to $69.2 \%$. Sensitivity rose to $96.7 \%$ using PROTOCOL III, specificity being of 79.3\%. Finally, PROTOCOL IV offered a sensitivity of $99.2 \%$, but specificity was of only $61 \%$. The sensitivity of the Van den Bergh and Piéret criteria [2] was of $79.5 \%$ for the 4 centres combined. Combined specificity was $96.9 \%$. There were no significant differences in the sensitivity rates between Leicester, Paris and Angers for the EFNS/PNS criteria, with any protocol. Sensitivity and specificity of Van den Bergh and Piéret's criteria were also equivalent in all 4 centres, and comparable to those of the EFNS/PNS with PROTOCOL I, indicating that distal CMAP duration prolongation as an additional parameter to the electrophysiological criteria did not have much impact. American Academy of 
Neurology (AAN) electrophysiological criteria for CIDP [8] showed a sensitivity of $45.7 \%$ in our 151 patients, and specificity of $100 \%$ versus controls. Again, the figures were comparable in all 4 centres.

The EFNS/PNS criteria, used with PROTOCOL I, offered significant improvement in diagnostic sensitivity compared to Koski et al.'s criteria. We evaluated both these criteria on 101 of our patients, excluding those with serum paraprotein and with a clinical diagnosis of Lewis-Sumner syndrome, which Koski et al. distinguished from CIDP, [5] and excluding the patients from Brussels, for whom distal CMAP duration data was not available. Considering these 101 patients, 65 (25/39 [64.1\%] from Leicester, 26/40 [65\%] from Paris and 14/22 [63.6\%] from Angers) fulfilled Koski et al.'s criteria, in comparison to 85 (33/39 [84.6\%] from Leicester, 33/40 [82.5\%] from Paris and 19/22 [86.4\%] from Angers), who met EFNS/PNS criteria with PROTOCOL I (Mc Nemar's Test; $\mathrm{P}=0.000002)$. Forty-one of the 101 patients fulfilled Koski et al.'s clinical criteria, and 51 fulfilled the electrophysiological criteria. All 101 cases met EFNS/PNS clinical criteria for typical or atypical CIDP, and 85 fulfilled the electrodiagnostic requirements. The 20 additional diagnoses achieved with the EFNS/PNS criteria were those which did not meet either clinical or electrophysiological criteria described by Koski et al., but met the EFNS/PNS clinical criteria for atypical CIDP and its electrophysiological requirements.

Compared to AAN criteria which only identified 56 of 123 cases from the abovementioned 3 centres, EFNS/PNS criteria allowed diagnosing 100 of 123 these patients (Mc Nemar's Test: P < 0.000001). With PROTOCOL I, EFNS/PNS criteria were of comparable specificity to those of Koski et al. $(\mathrm{P}=0.11)$. 
Table 4: Sensitivity of Koski et al.'s criteria [5], EFNS/PNS electrodiagnostic criteria [1], Van den Bergh and Piéret electrodiagnostic criteria [2], AAN electrodiagnostic criteria [8] in patients with a clinical diagnosis of CIDP

\begin{tabular}{|c|c|c|c|c|c|c|c|c|}
\hline & $\begin{array}{l}\text { Number of } \\
\text { patients with a } \\
\text { clinical } \\
\text { diagnosis of } \\
\text { CIDP } \\
\text { (EFNS/PNS) }\end{array}$ & $\begin{array}{l}\text { Sensitivity } \\
\text { of } \\
\text { Koski et } \\
\text { al.'s } \\
\text { criteria }\end{array}$ & $\begin{array}{l}\text { Sensitivity of } \\
\text { EFNS/PNS } \\
\text { electrodiagostic } \\
\text { criteria } \\
\text { (PROTOCOL } \\
\text { I) }\end{array}$ & $\begin{array}{l}\text { Sensitivity of } \\
\text { EFNS/PNS } \\
\text { electrodiagnostic } \\
\text { criteria } \\
\text { (PROTOCOL } \\
\text { II) }\end{array}$ & $\begin{array}{l}\text { Sensitivity of } \\
\text { EFNS/PNS } \\
\text { electrodiagnostic } \\
\text { criteria } \\
\text { (PROTOCOL } \\
\text { III) }\end{array}$ & $\begin{array}{l}\text { Sensitivity of } \\
\text { EFNS/PNS } \\
\text { electrodiagnostic } \\
\text { criteria } \\
\text { (PROTOCOL } \\
\text { IV) }\end{array}$ & $\begin{array}{l}\text { Sensitivity of } \\
\text { Van den Bergh } \\
\text { and Piéret's } \\
\text { electrodiagnostic } \\
\text { criteria } \\
\text { (PROTOCOL I) }\end{array}$ & $\begin{array}{l}\text { Sensitivity of } \\
\text { AAN } \\
\text { electrodiagnostic } \\
\text { criteria by distal } \\
\text { motor nerve study }\end{array}$ \\
\hline $\begin{array}{l}\text { Leicester, } \\
\text { U.K. }\end{array}$ & 54 & $\begin{array}{l}25 / 39 \\
{[64.1 \%]}\end{array}$ & $44 / 54$ [81.5\%] & $53 / 54$ [98.1\%] & $52 / 54[96.3 \%]$ & $54 / 54$ [100\%] & $43 / 54[79.6 \%]$ & $24 / 54$ [44.4\%] \\
\hline $\begin{array}{l}\text { Angers, } \\
\text { France. }\end{array}$ & 29 & $\begin{array}{l}14 / 22 \\
{[63.6 \%]}\end{array}$ & $23 / 29$ [79.3\%] & $27 / 29[93.1 \%]$ & $27 / 29[93.1 \%]$ & $28 / 29[96.6 \%]$ & $23 / 29[79.3 \%]$ & $11 / 29$ [37.9\%] \\
\hline $\begin{array}{l}\text { Brussels, } \\
\text { Belgium. }\end{array}$ & 28 & $\begin{array}{l}15 / 26 \\
{[57.7 \%]}\end{array}$ & - & - & - & - & $21 / 28[75 \%]$ & $11 / 28[39.3 \%]$ \\
\hline TOTAL & 151 & $\begin{array}{l}80 / 127 \\
{[63 \%]}\end{array}$ & $\begin{array}{l}100 / 123 \\
{[81.3 \%]}\end{array}$ & $119 / 123[96.7 \%]$ & $119 / 123[96.7 \%]$ & $122 / 123[99.2 \%]$ & $120 / 151[79.5 \%]$ & $69 / 151[45.7 \%]$ \\
\hline
\end{tabular}


Table 5: Specificity of Koski et al.'s criteria, EFNS/PNS criteria with various diagnostic subcategories, Van den Bergh and Piéret's criteria and AAN criteria: Controls with a clinical diagnosis of chronic distal sensory or sensori-motor neuropathy.

\begin{tabular}{|c|c|c|c|c|c|c|c|c|}
\hline & $\begin{array}{l}\text { Number of } \\
\text { Controls }\end{array}$ & $\begin{array}{l}\text { Specificity } \\
\text { of Koski et } \\
\text { al.'s } \\
\text { criteria }\end{array}$ & $\begin{array}{l}\text { Specificity } \\
\text { of } \\
\text { EFNS/PNS } \\
\text { criteria by } \\
\text { PROTOCOL } \\
\text { I }\end{array}$ & $\begin{array}{l}\text { Specificity } \\
\text { of } \\
\text { EFNS/PNS } \\
\text { criteria by } \\
\text { PROTOCOL } \\
\text { II }\end{array}$ & $\begin{array}{l}\text { Specificity } \\
\text { of } \\
\text { EFNS/PNS } \\
\text { criteria by } \\
\text { PROTOCOL } \\
\text { III }\end{array}$ & $\begin{array}{l}\text { Specificity } \\
\text { of } \\
\text { EFNS/PNS } \\
\text { criteria by } \\
\text { PROTOCOL } \\
\text { IV }\end{array}$ & $\begin{array}{l}\text { Specificity } \\
\text { of Van den } \\
\text { Bergh and } \\
\text { Piéret's } \\
\text { criteria by } \\
\text { PROTOCOL } \\
\text { I }\end{array}$ & $\begin{array}{l}\text { Specificity } \\
\text { of AAN } \\
\text { criteria by } \\
\text { distal } \\
\text { study of up } \\
\text { to } 8 \text { nerves }\end{array}$ \\
\hline $\begin{array}{l}\text { Leicester, } \\
\text { U.K. }\end{array}$ & $\begin{array}{l}95 \\
\text { (only } 47 \text { with } \\
\text { PROTOCOLS } \\
\text { III and IV) }\end{array}$ & $100 \%[0]$ & $96.8 \%[3]$ & $69.5 \%$ [29] & $80.9 \%$ [9] & $59.6 \%$ [19] & $96.8 \%[3]$ & $100 \%[0]$ \\
\hline $\begin{array}{l}\text { Paris, } \\
\text { France. }\end{array}$ & 35 & $100 \%[0]$ & $94.3 \%$ [2] & $68.6 \%$ [11] & $77.1 \%$ [8] & $62.9 \%$ [13] & $94.3 \%$ [2] & $100 \%[0]$ \\
\hline $\begin{array}{l}\text { Brussels, } \\
\text { Belgium. }\end{array}$ & $\begin{array}{l}32 \\
\text { (only } 22 \text { for } \\
\text { Koski et al.'s } \\
\text { criteria) }\end{array}$ & $95.5 \%[1]$ & - & - & - & - & $100 \%[0]$ & $100 \%[0]$ \\
\hline TOTAL & $\begin{array}{l}162 \\
\text { ( } 82 \text { with more } \\
\text { extensive } \\
\text { studies) }\end{array}$ & $99.3 \%[1]$ & $96.1 \%[5]$ & $71.5 \%$ [37] & $79.3 \%$ [17] & $61 \%$ [32] & $96.9 \%$ [5] & $100 \%[0]$ \\
\hline
\end{tabular}

N.B. Patients from Brussels all underwent only unilateral right-sided distal studies and were not evaluated for distal CMAP duration.

PROTOCOL I = unilateral right-sided distal study of 4 motor nerves \{“definite CIDP" + "probable CIDP"\}

PROTOCOL II = unilateral right-sided distal study of 4 motor nerves \{ “definite CIDP” + "probable CIDP” + "possible

CIDP'\}

PROTOCOL III = proximal and distal study of 5 to 8 motor nerves, with proximal studies for at least 2 arm nerves \{"definite CIDP" + "probable CIDP"'

PROTOCOL IV= proximal and distal study of 5 to 8 motor nerves, with proximal studies for at least 2 arm nerves

\{“definite CIDP" + probable CIDP" + "possible CIDP"\} 


\section{Discussion.}

The diagnostic criteria by Koski et al. [5] offer the potential convenience and advantage of combining clinical and electrophysiological components, either of which suffices for a positive diagnosis of CIDP. These criteria were derived from classification, performed through generation of an expert consensus diagnosis (the "gold standard") and regression tree analysis of 150 patients, amongst which 58 had CIDP, and later validated in 48 other CIDP subjects. However, they exclude patients with Lewis-Sumner syndrome, as well as those with associated monoclonal gammopathy.

Although shown to be of high sensitivity and specificity in one CIDP patient population, [7] the electrophysiological criteria for CIDP defined by the EFNS/PNS Guidelines [1] have not been evaluated more widely in different patient populations. Importantly, the issue of their specificity, when including cases of "possible CIDP" has remained uncertain. Furthermore, although utilized within other criteria, $[9,11]$ and shown to be reliable with appropriate cut-offs for conduction block and temporal dispersion, [10] the usefulness of proximal studies has not been previously assessed with the EFNS/PNS criteria.

The present study demonstrates high sensitivity of the EFNS/PNS criteria with unilateral 4-nerve distal segment studies. Our findings show the added benefit of these criteria in comparison with those of Koski et al. (Mc Nemar's Test; $\mathrm{P}=0.000002$ ). The specificity was high overall with PROTOCOL I, and individually in the two centres where controls were recruited. As compared to the AAN criteria, the 
EFNS/PNS criteria allowed a significant additional number of patients to be diagnosed (Mc Nemar's Test; $\mathrm{p}<0.00001$, for both PROTOCOLS I and III). We found that more extensive nerve conduction studies (PROTOCOL III) improved diagnostic sensitivity from $80.6 \%$ to $98.7 \%$. However, this was achieved with decline in specificity from $96.1 \%$ to $79.3 \%$. Use of the "possible CIDP" subcategory improved the sensitivity of unilateral 4-nerve distal studies (PROTOCOL II) by $15.4 \%$ but unacceptably worsened specificity by $24.6 \%$. With more extensive nerve conductions and inclusion of cases of "possible CIDP” (PROTOCOL IV), additional gain in sensitivity was minimal $(2.5 \%)$, but loss of specificity considerable (further decline by $18.3 \%)$.

The recommended unilateral distal segment 4 nerve study protocol allowed in our patients the identification of over $80 \%$ of cases with the EFNS/PNS criteria, with a very high specificity, comparable to that of the AAN criteria. Likely reasons for this high sensitivity are the need for abnormality of a single parameter to be present in 2 nerves, in contrast to the AAN criteria, which require abnormalities of three different parameters, notwithstanding the fact that the EFNS/PNS criteria set higher abnormality thresholds (e.g. for motor nerve conduction velocity, distal motor latency and conduction block), thereby allowing preservation of specificity. The usefulness of 8 nerve distal segment studies and proximal upper limb stimulations has been demonstrated previously with other electrodiagnostic criteria for CIDP [3, 9]. High specificity of the proximal component of upper limb nerve conductions has also been shown [10], at previously advised cut-offs $[9,12]$. Our present study confirms the utility and reliability of these procedures with the EFNS/PNS criteria. Although reduced, specificity remains high at around $80 \%$ with such more extensive studies, 
using PROTOCOL III. One reason for this relative reduction in specificity despite improved sensitivity with increase in number of tested nerves, was the severity of axonal loss in some controls, accompanied by significant motor conduction velocity reduction, distal motor latency prolongation or temporal dispersion, these parameters having standardized cut-off values independent of CMAP. Furthermore, the specificity of temporal dispersion $>30 \%$ at axilla and Erb's point, is suboptimal, as previously demonstrated, [10] and this also contributed to false positives.

To our knowledge, a single previous study demonstrated the high diagnostic value of the EFNS/PNS criteria, reporting a sensitivity of $97 \%$. [7] The extensiveness of the electrophysiology was uncertain as described in this analysis, in particular regarding uni- or bilaterality of the studies. This could explain the higher sensitivity to that we have found with PROTOCOL I. Furthermore, only symmetrical presentations were included in that study, and this may also have have increased the sensitivity of the EFNS/PNS criteria, as the majority of patients with Lewis-Sumner syndrome do not have diffuse electrophysiological demyelination. [13] Finally, this study also excluded cases of CIDP with concurrent illnesses such as diabetes, which may have impacted on the results.

Van den Bergh and Piéret's original criteria [2] independently offered comparable sensitivity to those of the EFNS/PNS with PROTOCOL I, in our patients. These results suggest that use of distal CMAP duration prolongation may not contribute to substantially increasing diagnostic yield within the criteria. Considering only cases from Leicester, Paris and Angers, distal CMAP duration prolongation of $>9$ milliseconds in any motor nerve, contributed to only one additional diagnosis (100 vs. 
99) from a total of 123 patients, as all the other patients positive for this criterion also showed demyelinating range changes for other parameters. The high diagnostic sensitivity for Van den Bergh and Piéret's criteria was initially demonstrated in the original series (75\%). [2] This was also shown in a study of 32 patients from a single centre $(71.8 \%),[14]$ which compared these criteria to those of the Inflammatory Neuropathy Cause and Treatment group criteria (sensitivity of 68.7\%) [9] and those of the AAN (sensitivity of 48.6\%). [6] The current findings are otherwise also in keeping with those of another previous analysis, from one of our 4 centres, which found distal CMAP duration prolongation in only 6 of 20 (30\%) CIDP patients [10].

The comparative values of diagnostic criteria for CIDP have rarely been studied in a large number of patients from different centres. Previous studies have highlighted the poor sensitivity of older criteria such as those of the AAN. $[1-4,8]$ Our findings are limited by the difficulties in comparing a set of criteria requiring fulfilment of either clinical or electrophysiological components (Koski et al.'s), [5] and of another requiring mandatory electrophysiology (EFNS/PNS). [1] The methodology was further complicated by the different clinical definitions for "CIDP" as used within the 2 different sets. However, comparison of the 101 patients from our study, acceptable for evaluation according to the definitions of both sets (excluding cases of LewisSumner syndrome and with associated monoclonal gammopathy), suggests the EFNS/PNS criteria are more sensitive than those of Koski et al. while offering similar high specificity. In our current analysis, only 51 of the 101 patients met Koski et al.'s electrophysiological criteria while 85 met those of the EFNS/PNS. Koski et al.'s clinical criteria fail to pick up atypical cases such as those with distal or asymmetrical involvement or patients without four limb motor weakness. Considering that 
diagnostic difficulty may precisely arise in such patients in practice, this is a limitation to their utility. Illustrating this point, only 41 of the 101 patients met Koski et al.'s clinical criteria, while they all fulfilled EFNS/PNS criteria for typical or atypical CIDP. In addition, Koski et al.'s electrophysiological criteria require at least $75 \%$ of excitable motor nerves and $>50 \%$ of these exhibiting either abnormal distal latency, conduction velocity, or F latency. This can be problematic in cases with severe secondary axonal degeneration, or in patients tested for an even number of nerves. Furthermore, we here compared EFNS/PNS criteria with PROTOCOL I, i.e. using 4 studied nerves, with Koski et al.'s criteria, using all available nerves, which artificially increased the sensitivity of the latter, as all our patients were evaluated for 4 nerves or more. Also, as proximal weakness in at least one limb was mandatory for fulfilment of Koski et al.'s clinical criteria, use of controls with distal sensory or sensori-motor neuropathy clearly increased the specificity of these criteria. Finally, the present results also demonstrate the possibility of usefully and reliably utilizing the EFNS/PNS criteria with more extensive nerve conduction studies, including proximal stimulations for upper limb nerves. The poor specificity of the "possible CIDP" electrodiagnostic subcategory found here, however suggests it should be used cautiously.

Competing interests: none declared. 


\section{References:}

1. Hughes RA, Bouche P, Cornblath DR, et al. European Federation of Neurological societies/Peripheral Nerve Society guideline on management of chronic inflammatory demyelinating polyneuropathy: report of a joint task force of the European Federation of Neurological Societies and the Peripheral Nerve Society. Eur J Neurol 2006;13:326-332.

2. Van den Bergh PYK, Piéret F. Electrodiagnostic criteria for acute and chronic inflammatory demyelinating polyradiculoneuropathy. Muscle Nerve 2004;29:565-574.

3. Rajabally YA, Jacob S, Hbahbih M. Optimizing the use of electrophysiology in the diagnosis of chronic inflammatory demyelinating polyneuropathy: a study of 20 cases. J Peripher Nerv Syst 2005;10:282-292.

4. Magda P, Latov N, Brannagan TH III , et al. Comparison of electrodiagnostic abnormalities and criteria in a cohort of patients with chronic inflammatory demyelinating polyneuropathy. Arch Neurol 2003;60:1755-1759.

5. Koski CL, Baumgarten M, Magder LS, et al. Derivation and validation of diagnostic criteria for chronic inflammatory demyelinating polyneuropathy. J Neurol Sci 2009;277:1-8.

6. Thaisetthawatkul P, Logigian EL, Herrmann DN. Dispersion of the distal compound muscle action potential as a diagnostic criterion for chronic inflammatory demyelinating polyneuropathy. Neurology 2002;59:1526-1532. 
7. Tackenberg B, Lünemann JD, Steinbrecher A, et al. Classifications and treatment responses in immune-mediated demyelinating polyneuropathy. Neurology 2007;68:1622-1629.

8. Ad Hoc Subcommittee of the American Academy of Neurology AIDS Taskforce. Research criteria for diagnosis of chronic inflammatory demyelinating polyneuropathy (CIDP). Neurology 1991;41:617-618.

9. Nicolas G, Maisonobe T, Le Forestier N, et al. Proposed revised electrophysiological criteria for chronic inflammatory demyelinating polyradiculoneuropathy. Muscle Nerve 2002;25:26-30.

10. Rajabally YA, Jacob S. Proximal nerve conduction studies in chronic inflammatory demyelinating polyneuropathy. Clin Neurophysiol 2006;117:20792084.

11. Hughes R, Bensa S, Willison H, et al.; Inflammatory Neuropathy Cause and Treatment (INCAT) Group. Randomized controlled trial of intravenous immunoglobulin versus oral prednisolone in chronic inflammatory demyelinating polyradiculoneuropathy. Ann Neurol 2001;50:195-201.

12. Oh SJ, Kim DE, Kuruoglu HR. What is the best diagnostic index of conduction block and temporal dispersion? Muscle Nerve 1994;17:489-493.

13. Rajabally YA, Chavada G. Lewis-Sumner syndrome of pure upper-limb onset: diagnostic, prognostic and therapeutic features. Muscle Nerve 2009;39:206-220. 
14. Cocito D, Chiò A, Tavella A, et al. Treatment response and electrophysiological criteria in chronic inflammatory demyelinating polyneuropathy. Eur J Neurol 2005;13:666-672. 